\title{
Preliminary daytime seeing monitoring at Dome A, Antarctica
}

\author{
Chong Pei ${ }^{1,2,3,4}$ Zhengyangg Li ${ }^{1,2,3}$ Hualin Chen ${ }^{1,2,3}$ \\ and Xiangyan Yuan ${ }^{1,2}$ \\ ${ }^{1}$ National Astronomical Observatories/Nanjing Institute of Astronomical Optics \& Technology, \\ Chinese Academy of Sciences, Nanjing 210042, China \\ ${ }^{2}$ Key Laboratory of Astronomical Optics \& Technology, Nanjing Institute of Astronomical \\ Optics \& Technology, Chinese Academy of Sciences, Nanjing 210042, China \\ ${ }^{3}$ Graduate University of Chinese Academy of Sciences, Beijing 100049, China \\ ${ }^{4}$ email: cpei@niaot.ac.cn
}

\begin{abstract}
Sites on Antarctic plateau have unique atmospheric properties that make them better than any mid-latitude sites as observatory locations. From site testing measurements over 4 years on Dome A carried out by the Chinese Center for Antarctic Astronomy, we can reasonably predict that Dome A is as good as or even better than Dome $\mathrm{C}$, which has been proved to be the best astronomical site by now, and suitable for high angular resolution observations. Seeing monitoring is necessary for planning large scale ground-based optical astronomical telescopes. In 2012, the 28th Chinese Antarctic Scientific Expedition carried out preliminary daytime seeing monitoring using a Differential Image Motion Monitor (DIMM) placed at a height of $3.5 \mathrm{~m}$. The median seeing was found to be $0.8^{\prime \prime}$. This will be the foundation of future research that obtains comprehensive and long-period monitoring of the site's optical parameters.
\end{abstract}

Keywords. Dome A, Seeing, Isoplanatic angle.

\section{Theory}

Fried (1966) introduced a parameter of atmospheric coherence length $r_{0}$ (Roddier (1981)), and he derived the relation of $\varepsilon_{F W H M}=0.98 \lambda / r_{0}$, generally expressed in units of arcseconds. A DIMM measures the variance of differential image motion which is related to wavelength, telescope diameter and Fried parameter $r_{0}$ as: $\sigma_{d}^{2}=K \lambda^{2} r_{0}^{-5 / 3} D^{-1 / 3}$ (Jean \& Casiana (1995)), so $\varepsilon_{0}=0.98\left(\sigma_{d}^{2}\right)^{3 / 5}(D / \lambda)^{1 / 5} K^{-3 / 5}$. The isoplanatic angle can be estimated from the fluctuations of stellar flux received by an annular sub-aperture of this site testing instrument as $\theta_{0}^{-5 / 3}=A \sigma_{I}^{2} \cos z^{-8 / 3}$.

\section{Instrument}

A $35 \mathrm{~cm}$ Celestron commercial telescope tube with focal length of $3910 \mathrm{~mm}$ matched with a pupil mask and CCD camera was mounted on the first of three Antarctic Survey Telescopes (AST3-1) which has high pointing and tracking accuracy and could work at the very low temperature at Dome A. The special software with graphic user interface is designed for displaying real-time images, computing results and running status and longdistance control. The Dome A seeing monitoring system are formed by these hardware and software, shown in Figure 1. Three glasses with wedge angles of $50^{\prime \prime}$ coated with ITO film to keep the frost and ice away from the surface in winter have been located on the mask attached to the telescope entrance pupil. 


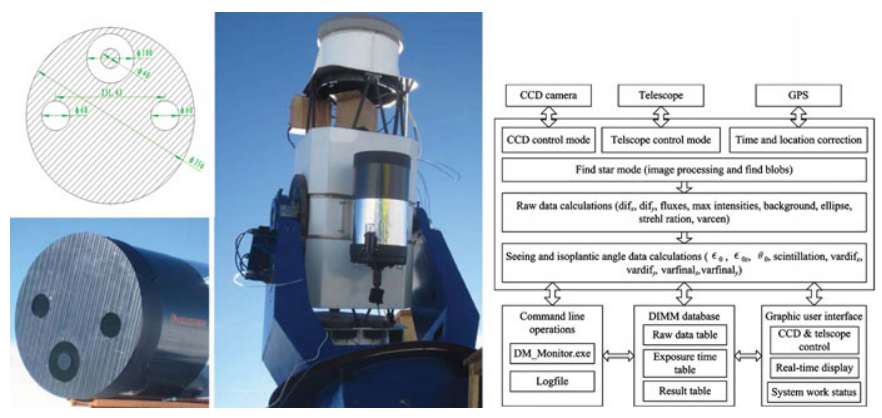

Figure 1. Left: the hardware of the Dome A seeing monitoring system. Right: organisation of the software of the Dome A seeing monitoring system.
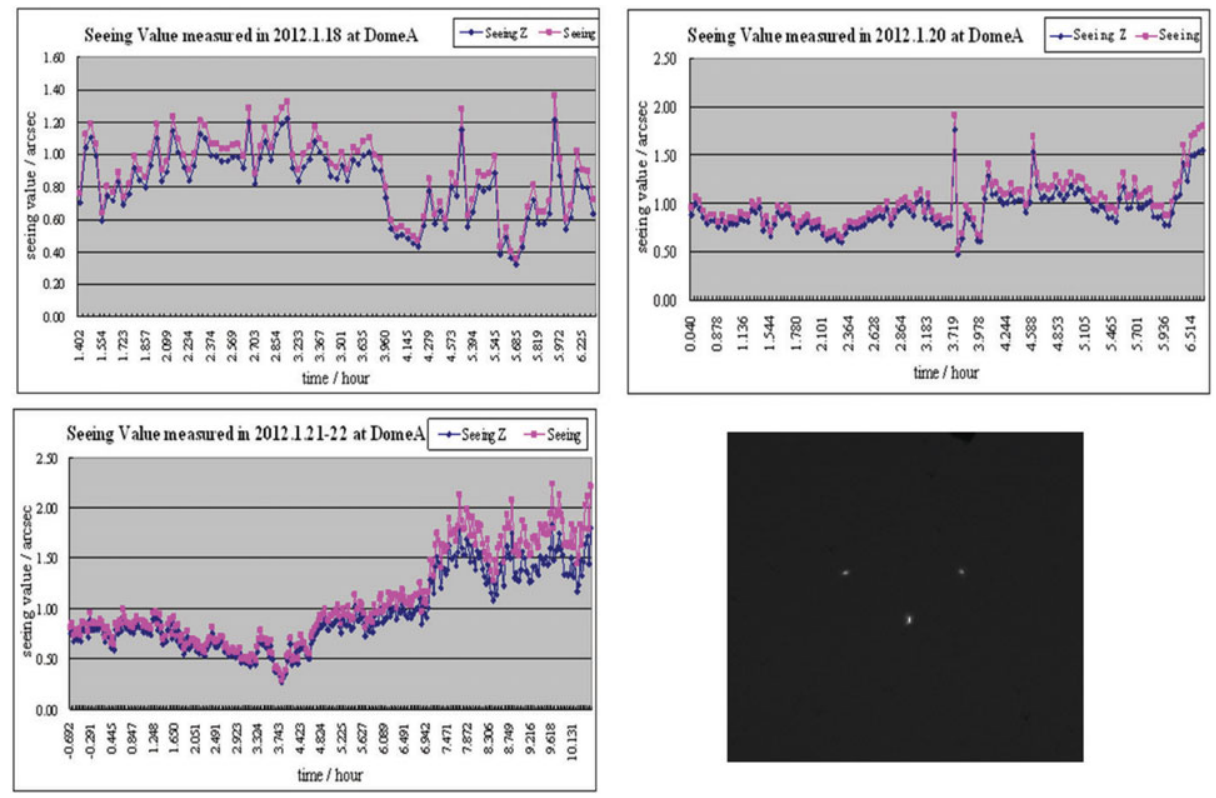

Figure 2. The results of seeing measurement and the image captured by the CCD with an exposure time of 5 millisecond.

\section{Result}

The successful operation of this seeing monitoring system enables us firstly to obtain the preliminary daytime seeing results for three days at Dome A by observing Canopus (-0.72 mag). The results are plotted in the following charts. In Figure 2, the upper left one is the result obtained during 01:22 to 06:20 on January 18, which shows seeing values centralised in the range of $0.87^{\prime \prime} \sim 0.97^{\prime \prime}$ and accounts for $22 \%$. The upper right one is the result obtained during 00:02 to 07:18 on January 20, which shows seeing values centralised in the range of $0.72^{\prime \prime} \sim 0.83^{\prime \prime}$ and accounts for $27 \%$. The lower left one is the result taken during 23:18 on January 21 to 10:59 on January 22, and shows seeing values centralised in the range of $0.7^{\prime \prime} \sim 0.8^{\prime \prime}$ and accounts for $19.7 \%$.

\section{References}

Roddier, F. 1981, Progress in optics, 19, 281

Jean, V. \& Casiana, M. 1995, PASP, 107, 265 